\title{
Vancomycin-resistant Enterococcus domination of intestinal microbiota is enabled by antibiotic treatment in mice and precedes bloodstream invasion in humans
}

\author{
Carles Ubeda, ${ }^{1,2}$ Ying Taur, ${ }^{1}$ Robert R. Jenq, ${ }^{3}$ Michele J. Equinda,, ${ }^{1,2}$ Tammy Son, ${ }^{3}$ \\ Miriam Samstein, ${ }^{1,2}$ Agnes Viale, ${ }^{4}$ Nicholas D. Socci, ${ }^{5}$ Marcel R.M. van den Brink, ${ }^{2,3}$ \\ Mini Kamboj, ${ }^{1}$ and Eric G. Pamer ${ }^{1,2}$
}

\begin{abstract}
${ }^{1}$ Infectious Diseases Service, Department of Medicine, Memorial Sloan-Kettering Cancer Center, New York, New York, USA. ${ }^{2}$ Immunology Program, Sloan-Kettering Institute, New York, New York, USA. ${ }^{3}$ Bone Marrow Transplant Service, Department of Medicine, Memorial Sloan-Kettering Cancer Center, New York, New York, USA. ${ }^{4}$ Genomics Core Laboratory and ${ }^{5}$ Computational Biology Center, Sloan-Kettering Institute, New York, New York, USA.
\end{abstract}

\begin{abstract}
Bloodstream infection by highly antibiotic-resistant bacteria, such as vancomycin-resistant Enterococcus (VRE), is a growing clinical problem that increasingly defies medical intervention. Identifying patients at high risk for bacterial sepsis remains an important clinical challenge. Recent studies have shown that antibiotics can alter microbial diversity in the intestine. Here, we characterized these effects using 16s rDNA pyrosequencing and demonstrated that antibiotic treatment of mice enabled exogenously administered VRE to efficiently and nearly completely displace the normal microbiota of the small and large intestine. In the clinical setting, we found that intestinal domination by VRE preceded bloodstream infection in patients undergoing allogeneic hematopoietic stem cell transplantation. Our results demonstrate that antibiotics perturb the normal commensal microbiota and set the stage for intestinal domination by bacteria associated with hospital-acquired infections. Thus, high-throughput DNA sequencing of the intestinal microbiota could identify patients at high risk of developing bacterial sepsis.
\end{abstract}

\section{Introduction}

Antibiotic-resistant bacteria are an increasing problem in hospitalized patients. Methicillin-resistant Staphylococcus aureus (MRSA), Acinetobacter species, Pseudomonas aeruginosa, vancomycin-resistant Enterococcus faecium (VRE), and Enterobacteriaceae species such as Escherichia coli or carbapenem-resistant Klebsiella pneumoniae represent some of the major highly resistant organisms that cause infections in hospitalized patients (1-3). Infections with antibiotic-resistant bacteria generally begin with colonization of mucosal surfaces, in particular the intestinal epithelium. Mammalian gastrointestinal mucosal surfaces are inhabited by microbial populations, which, in aggregate, are referred to as the microbiota (4-7). Using cultivationindependent molecular techniques based on 16s rRNA gene sequencing, recent studies investigating commensal microbiota identified more than 1,000 different bacterial taxa in intestinal samples (8). Most of these different taxa belong to 2 phyla, Firmicutes and Bacteroidetes; a few belong to the phyla Actinobacteria, Proteobacteria, Verrucomicrobia, and Fusobacteria $(4,8,9)$. These commensal bacteria play important roles in food digestion (10), regulation of fat storage (11), stimulation of intestinal angiogenesis (12), protection against epithelial cell injury (13), development of the immune system (14), and resistance to colonization by pathogens (15).

Recent studies have shed light on mechanisms of microbiotamediated protection against pathogenic microbes and how antibiotic-mediated depletion of the microbiota increases colonization with highly antibiotic-resistant microorganisms $(16,17)$. Components of the microbiota, such as LPS, lipoteichoic acid, flagellin, or

Conflict of interest: The authors have declared that no conflict of interest exists. Citation for this article: J Clin Invest. 2010;120(12):4332-4341. doi:10.1172/JCI43918. muramyl dipeptide are recognized by Toll-like and Nod receptors expressed by intestinal epithelial and Paneth cells (18-21). Stimulation of these receptors in the small intestine triggers production and secretion of antimicrobial proteins, including antimicrobial peptides, such as $\alpha$-defensins and cryptdins, and the C-type lectin Reg3 $\gamma$, which selectively kills Gram-positive bacteria (16, 22-26). Depletion of the microbiota with an antibiotic cocktail containing metronidazole, neomycin, and vancomycin (MNV) diminishes expression of Reg $3 \gamma$, thereby decreasing resistance to VRE colonization (17). Reg3 $\gamma$ expression and susceptibility to VRE colonization in antibiotic-treated mice is restored by administration of oral LPS or systemic flagellin, which enhances VRE killing $(17,27)$. Thus, the intestinal microbiota promotes resistance to infection with highly antibiotic-resistant bacteria by inducing the production of antimicrobial peptides by intestinal epithelial cells.

Recent studies have shown that antibiotic treatment alters intestinal microbial diversity $(8,28-33)$. Administration of broad-spectrum antibiotics, for example, changes bacterial populations in the murine large intestine, reducing the total number of bacteria and diminishing cytokine production in the intestine (33). Studies in humans treated with a short course of ciprofloxacin demonstrated that changes in the microbiota persisted after cessation of antibiotic treatment (8). It remains unclear, however, how antibiotic-induced changes in the intestinal microbiota affect the establishment, expansion, and persistence of highly antibiotic-resistant bacteria in the intestine. Furthermore, whether the density of intestinal colonization with antibiotic-resistant bacteria correlates with the risk of bloodstream invasion is also unclear.

We have characterized antibiotic-induced changes in the intestinal microbiota and the effect of these changes on susceptibility to 

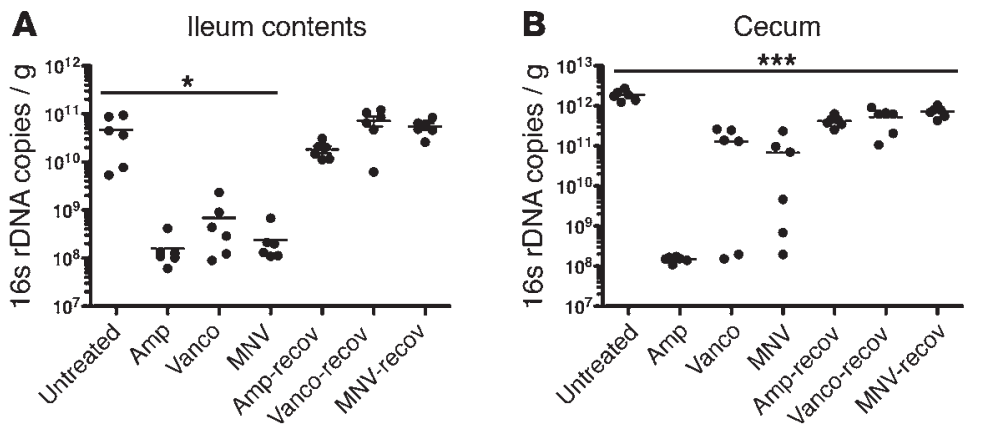

\begin{abstract}
Figure 1
Antibiotic treatment alters intestinal microbial density. The number of $16 \mathrm{~s}$ rDNA copies was determined by qPCR of (A) ileum or (B) cecum samples from untreated mice, mice treated with ampicillin (Amp), vancomycin (Vanco), or MNV, or mice 2 weeks after cessation (i.e., recovery [recov]) of antibiotic treatment with ampicillin, vancomycin, or MNV (6 mice per group). ${ }^{*} P<0.05,{ }^{\star \star *} P<0.001$ versus untreated, 1 -way ANOVA with Bonferroni correction.
\end{abstract}

intestinal colonization with VRE. Antibiotic treatment decreased the density of intestinal bacteria and dramatically altered the intestinal microbiota. Cessation of antibiotic treatment resulted in bacterial density increase; however, the recovered microbiota had decreased frequencies of bacteria belonging to the Bacteroidetes phylum and the Lactobacillaceae family and increased frequencies of bacteria belonging to the Clostridium and Enterococcus genera and the Enterobacteriaceae family. Whereas mice that did not receive antibiotics were resistant to colonization with VRE, antibiotic treatment enabled VRE to dominate the intestinal microbiota, reaching frequencies of $95 \%$ in the ileum and cecum. Once established, VRE remained a dominant member of the microbiota upon cessation of antibiotic treatment. Characterization of the microbiota colonizing patients undergoing allogeneic hematopoietic stem cell transplantation (allo-HSCT) revealed that antibiotic treatment of humans also enables VRE to dominate the intestinal microbiota, a detectable event that precedes VRE invasion of the bloodstream.

\section{Results}

Antibiotic effects on the intestinal microbiota. To study how antibioticinduced changes on the microbiota impact the intestinal colonization and persistence of highly antibiotic-resistant bacteria, we treated mice for 1 week with either MNV antibiotic cocktail; ampicillin, a commonly used antibiotic with a relatively narrow spectrum of activity; or vancomycin, an antibiotic that selectively kills Grampositive bacteria. Stool pellets, cecum contents, and ileum wall and contents were harvested in 6 mice immediately following the 7-day treatment, while another 6 mice were switched back to antibioticfree water for 2 weeks of recovery before harvesting the samples.

We first determined the effect of antibiotics on microbial density by quantitative PCR (qPCR) of bacterial 16s rRNA genes. All 3 antibiotic regimens decreased the number of $16 \mathrm{~s}$ rDNA copies in the ileum by a factor of approximately 100, whereas in the cecum, only ampicillin consistently reduced bacterial density (Figure 1, A and B). In 4 of 6 mice receiving vancomycin and 3 of 6 mice treated with $\mathrm{MNV}$, the quantity of 16 s genes was only reduced 10 -fold. At 2 weeks after ceasing antibiotic treatment, bacterial densities returned to normal in the ileum (Figure 1A). In the cecum, bacterial density increased after antibiotic cessation, although it was slightly lower than that of untreated mice $(P<0.001$; Figure $1 \mathrm{~B})$.

We next determined the composition of the intestinal microbiota during antibiotic treatment using the 454/Roche pyrosequencing platform. Because microbial and chloroplast DNA from ingested food contributes to 16 s rDNA sequences (33), we amplified and sequenced 16s rDNA sequences from our animal facility's murine feed. More than $90 \%$ of the $16 \mathrm{~s}$ rDNA sequences in murine feed were classified as Streptophyta, a Viridiplantae (data not shown).
Therefore, Streptophyta sequences, which represented up to $32 \%$ of the sequences (average, $5.75 \%$ ) in samples obtained from antibiotic-treated mice, were subtracted from our sequence analyses. After quality control (see Methods) and subtraction of Streptophyta sequences, we obtained a total of 116,542 sequences for this analysis. We obtained an average of 777 sequences per sample. Because of the low bacterial density, we could not obtain a PCR product of the 16s rRNA gene from samples of the ileum wall of mice during antibiotic treatment.

Consistent with previous studies (31), distinct bacterial communities inhabited the small and large intestine of untreated mice (Figure 2). The microbiota of the ileum contents and ileum wall was predominately populated by Lactobacillaceae and Erysipelotrichaceae (Figure 2 and Supplemental Figure 1; supplemental material available online with this article; doi:10.1172/JCI43918DS1), with frequencies varying between individual mice. The microbiota of the cecum and feces (Figure 2 and Supplemental Figure 1) consisted mostly of bacteria belonging to the Erysipelotrichaceae, Lactobacillaceae, Ruminococcaceae, Lachnospiraceae, and Coriobacteriaceae families; bacteria of the Clostridiales and Bacteroidales order; and other bacteria belonging to the Bacteroidetes phylum. Although the cecum and feces contained similar bacterial populations, their relative abundance differed (Supplemental Figure 1).

Antibiotic treatment had a dramatic effect on the microbiota of the small and large intestine (Figure 2 and Supplemental Figures 1 and 2). To more specifically define changes in the microbiota induced by antibiotic treatment, we plotted the increase and decrease of bacterial populations in a heatmap (Figure 3). All mice receiving ampicillin had similar changes in the microbiota of the ileum and cecum, with increased proportions of Streptococcaceae and decreased proportions of the other predominant families (Figure 2 and Figure 3A). Interestingly, both antibiotic treatments resulted in greater similarity among the microbiota of the ileum contents and cecum (Figure 2). As antibiotic treatment resulted in distension of the cecum, it is possible that cecal contents refluxed into the ileum.

In contrast to ampicillin treatment, the effect of vancomycin treatment on the microbiota was less uniform among individual mice. For example, 4 mice treated with vancomycin had dramatic expansion of Enterobacteriaceae in the ileum contents and cecum (Figure 2). Expansion of Enterobacteriaceae in these mice accounted for the relatively high bacterial density measured by qPCR of the 16 s rRNA gene (Figure 1B). The microbiota of mice treated with MNV underwent changes similar to those observed with vancomycin treatment (Supplemental Figure 1), with dramatic expansion of Enterobacteriaceae in 3 mice. Characterization of the microbiota 2 weeks after ceasing antibiotic treatment revealed that Erysipelotrichaceae and Lachnospiraceae became the most highly 
A

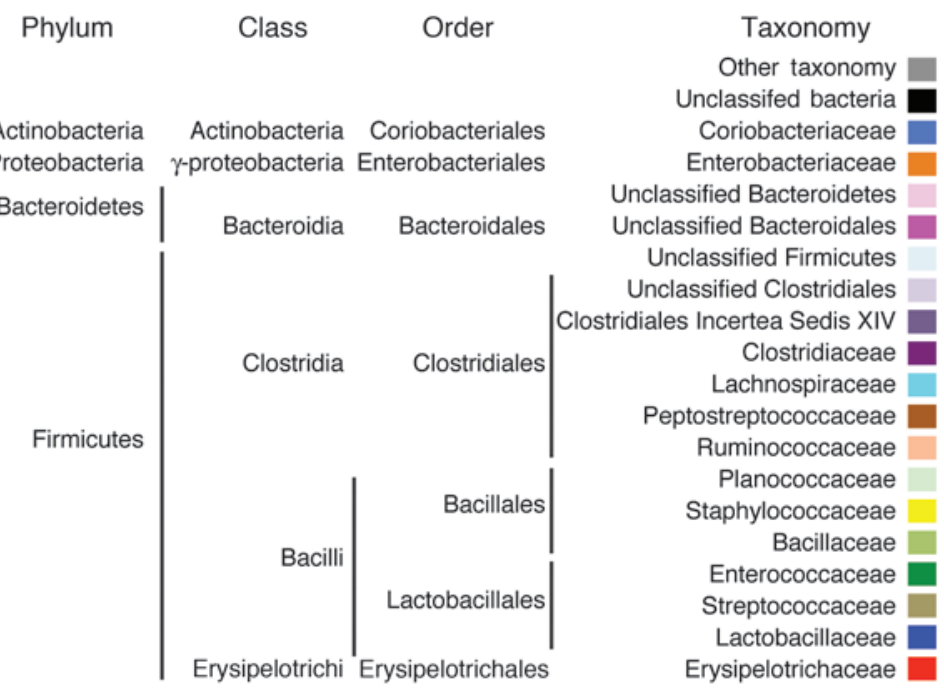
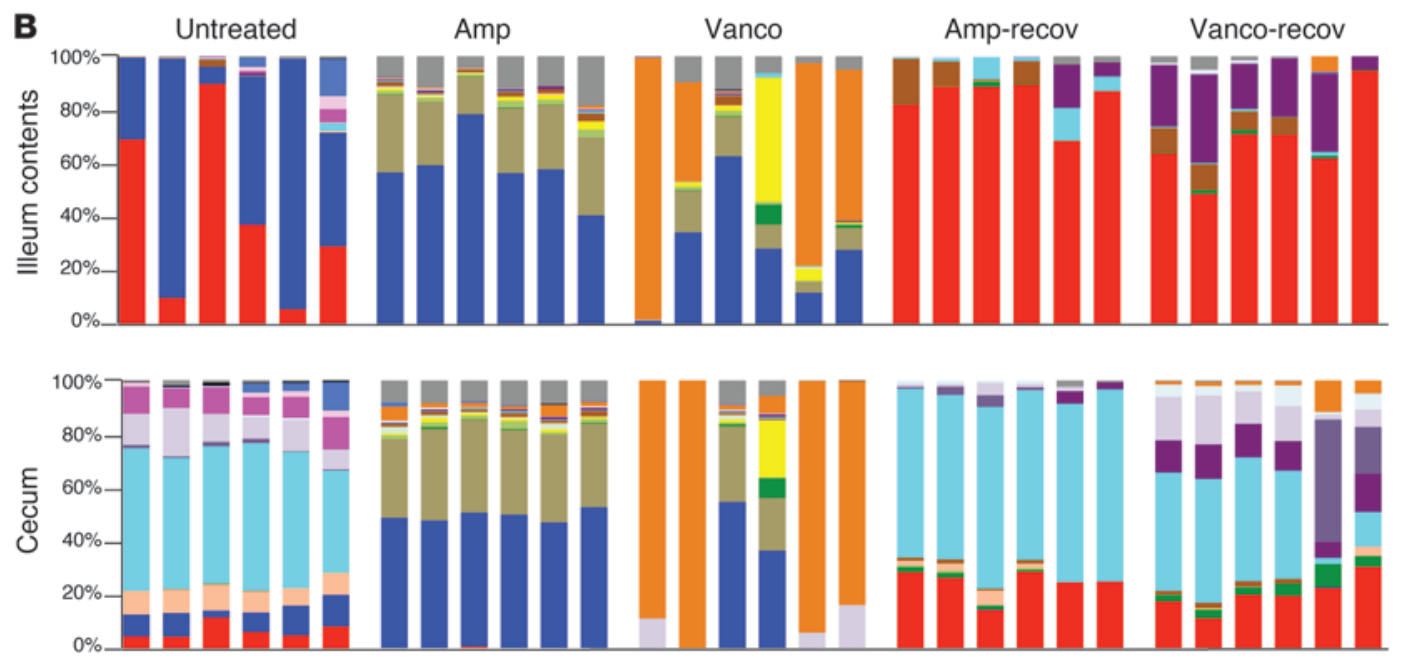

\section{Figure 2}

Antibiotic treatment alters the composition of the intestinal microbiota. (A) Color code for the most predominant bacterial taxa found in the murine intestine. (B) Phylogenetic classification of 16S rDNA frequencies in the ileum contents or cecum from samples collected from untreated mice, mice treated with ampicillin or vancomycin, or mice allowed to recover for 2 weeks from antibiotic treatment. Each bar represents the microbiota of an individual mouse.

represented bacterial families in the ileum and cecum, respectively (Figure 2). Remarkably, however, the frequencies of Lactobacillaceae and bacteria belonging to the Bacteroidetes phylum were markedly reduced in the ileum wall, cecum, and feces (Figure 3B).

At 2 weeks after ceasing vancomycin treatment, the frequency of Clostridiaceae, Enterococcaceae, and Enterobacteriaceae significantly increased in the cecum and feces (Figure 3B). An increase in Clostridiaceae was also observed in the ileum contents and wall (Figure 3B). Similarly, most of the mice treated with ampicillin or $\mathrm{MNV}$, at 2 weeks after ceasing antibiotics, had microbiota with higher frequencies of the genera Clostridium and Enterococcus (Figure 4). The family Enterobacteriaceae also increased in most of the mice after ceasing MNV treatment in the cecum and feces (Figure $4 \mathrm{~B})$. The increase in the frequency of bacteria belonging to the genera Clostridium and Enterococcus and the family Enterobacteriaceae is noteworthy because many of the major bacterial species causing hospital-acquired infections belong to these groups. Interest- ingly, the ileum wall of most of the untreated mice contained low numbers of Enterobacteriaceae (Figure 4A). Moreover, the most predominant Enterobacteriaceae operational taxonomic units (OTUs) found in mice treated with antibiotics were also present in the ileum wall of some of the untreated mice. This result suggests that the ileum wall may be the source of the Enterobacteriaceae that increased after antibiotic treatment.

Antibiotic effect on VRE intestinal colonization in mice. The previous results demonstrated that antibiotic-mediated disruption of the intestinal microbiota can result in domination of the intestinal microbiota by a very narrow range of microbial taxa, leading to markedly restricted diversity. Although previous studies have demonstrated that antibiotic treatment can lead to increased colonization of the intestine by $\operatorname{VRE}(17,34)$, to our knowledge, the prevalence of VRE relative to other intestinal microbes has not been previously determined. To address this question, untreated and ampicillin-treated mice were infected with $10^{8}$ CFUs of VRE. 
A

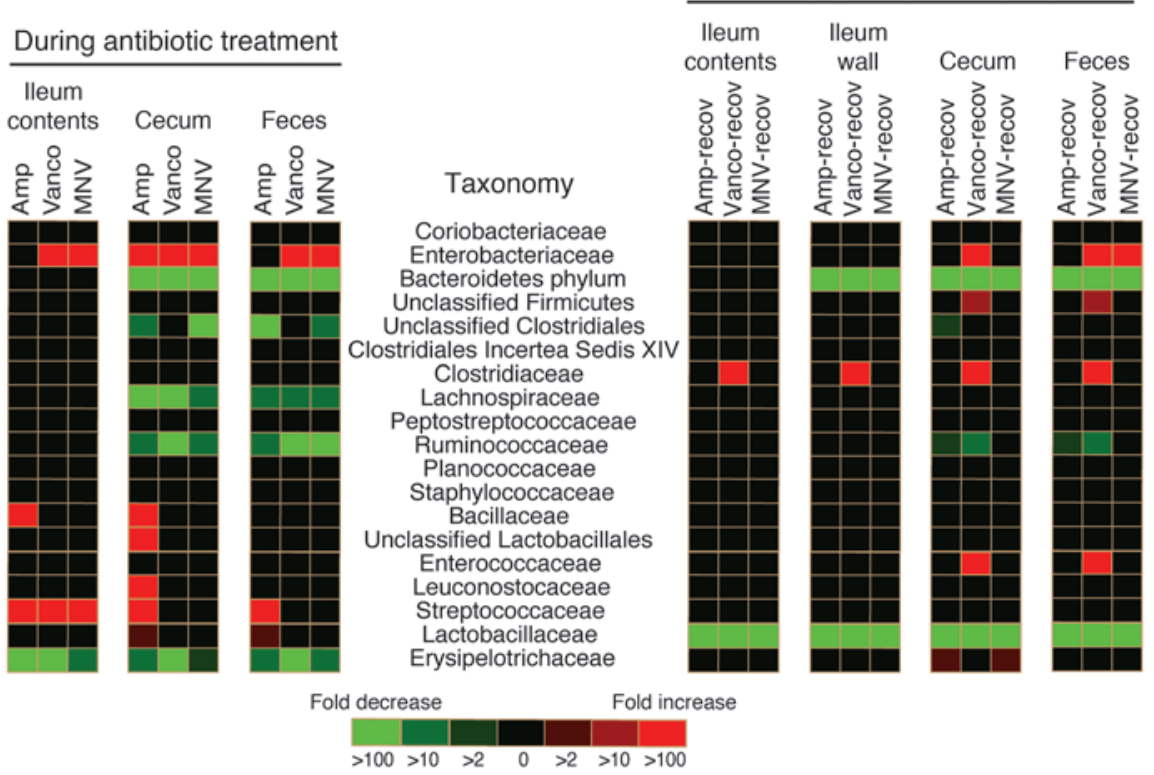

\section{Figure 3}

Statistically significant changes in intestinal bacterial populations observed upon antibiotic treatment. Heatmap showing statistically significant differences $(P<0.05)$ in intestinal bacterial populations between $(\mathbf{A})$ untreated mice and mice treated for 1 week with ampicillin, vancomycin, or MNV, or (B) untreated mice and mice allowed to recover for 2 weeks from antibiotic treatment. Colors in the key indicate the observed fold changes (red, increase; green, decrease).
Increased VRE density in the small intestine and cecum contents was detected in ampicillin-treated mice 1 day after infection (Figure 5A). While VRE density decreased 100-fold in untreated mice 8 days after infection, VRE frequencies markedly increased in mice continuously treated with ampicillin (Figure 5A). Pyrosequencing of $16 \mathrm{~s}$ rDNA revealed that VRE constituted nearly the entire microbiota in VRE-infected, ampicillin-treated mice (Figure 5B). To determine whether continuous exposure to antibiotic was necessary to maintain VRE colonization, we discontinued ampicillin treatment 1 day after VRE infection and quantified VRE at intervals afterward. Remarkably, 7 days after discontinuing ampicillin, VRE remained one of the most predominant bacterial species (Figure 5B). Since bacterial density was similar in untreated mice and ampicillin-treated, VRE-infected mice (Figure 5C), the density of VRE in antibiotic-treated mice approximated the density of the complex microbiota inhabiting the intestines of untreated mice. High levels of VRE were cultured from mice up to 2 months after cessation of ampicillin treatment (Figure 5A), which suggests that once VRE becomes established as a major component of the microbiota, either it acquires the ability to persist in the gut or the residual microbiota becomes more accommodating.

While the effects of antibiotic treatment on the intestinal microbiota extend beyond the course of antibiotic administration (Figure 2 and ref. 8), the duration of antibiotic effects on susceptibility to VRE colonization are not known. As a first step to address this issue, we determined the effect of ampicillin on the intestinal microbiota up to 8 weeks after ceasing antibiotic treatment. For

\section{Figure 4}

Antibiotic treatment promotes intestinal colonization by Clostridium, Enterococcus, and Enterobacteriaceae. Heatmap showing the frequencies of 16s rDNA sequences classified as Clostridium, Enterococcus, or Enterobacteriaceae in the small (A) or large (B) intestine of untreated mice or mice allowed to recover for 2 weeks after ampicillin, vancomycin, or MNV treatment. Colors in key indicate the frequency of each group of bacteria in each sample. Each row represents an individual mouse.
8 days, 4 groups of cohoused mice were treated with ampicillin; 1 mouse per group was euthanized 1, 2, 4, or 8 weeks after antibiotic withdrawal; and the microbiota of the cecum and ileum was characterized. Control mice for each group had not been treated with antibiotics. We first characterized the global effects of antibiotic treatment on the microbiota by quantifying similarities based on phylogenetic distances using UniFrac (35). We used unweighted UniFrac, which tabulates the presence or absence, but not the proportion, of different bacterial lineages, and principal coordinate analysis to cluster communities along orthogonal axes of maximal variance. Mice that did not receive antibiotic treatment were clustered by the 2 coordinates (PC1 and PC2) that explained most of
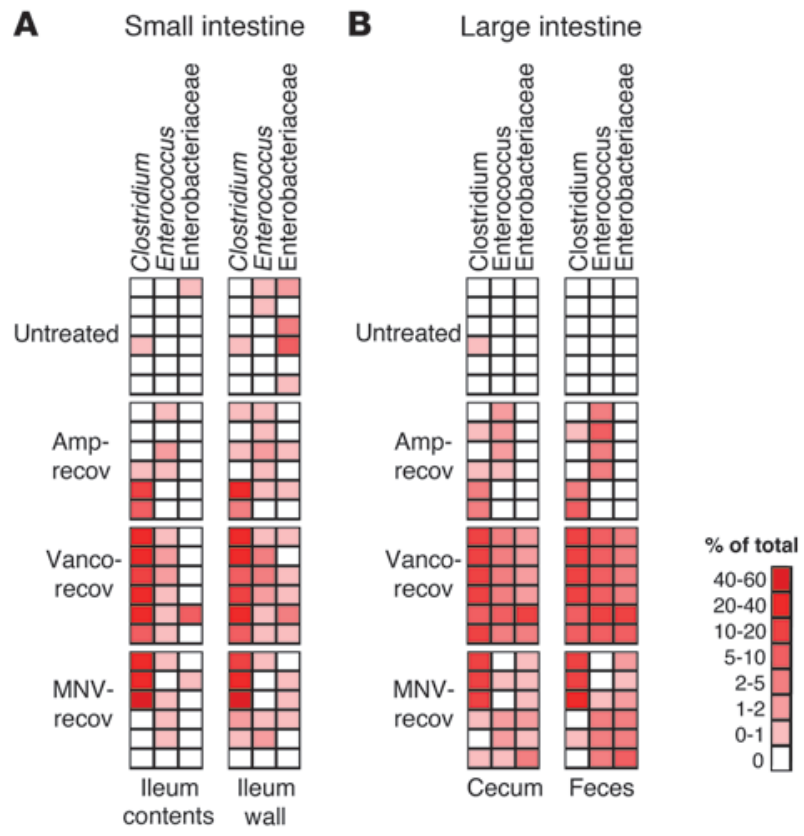

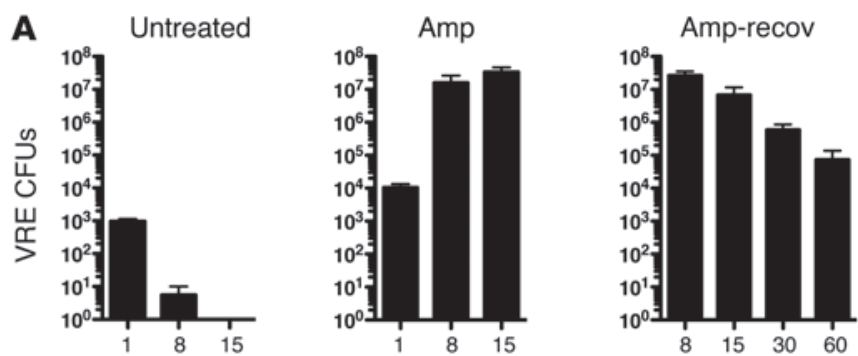

Small

Intestine
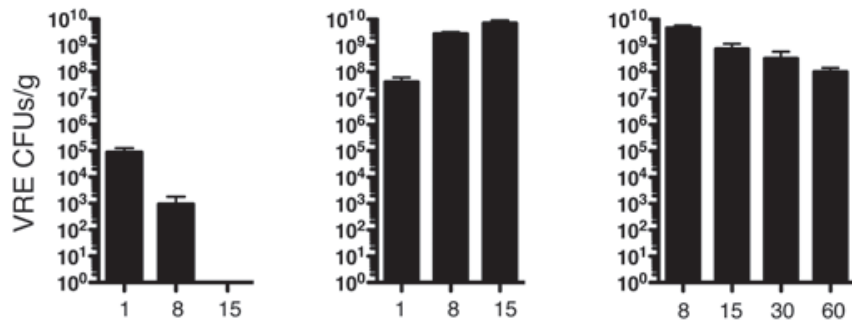

Time after VRE infection (d)

B Untreated Amp Amp + VRE
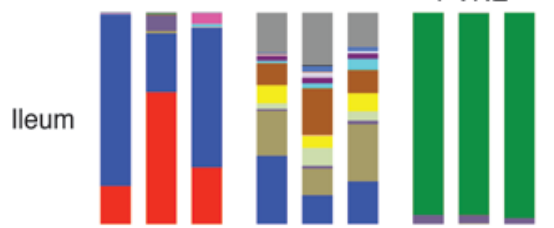
Amp-recov Amp-recov + VRE
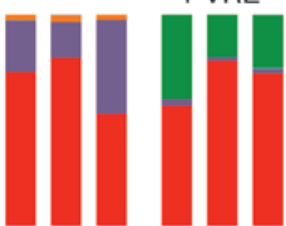

Cecum
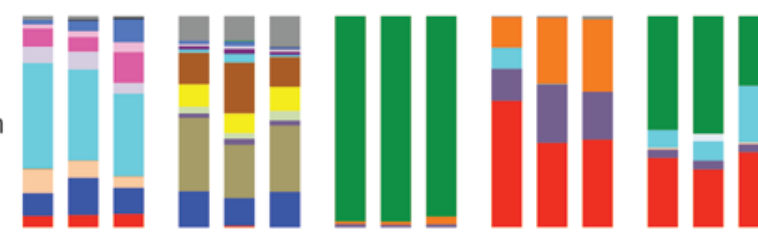

C

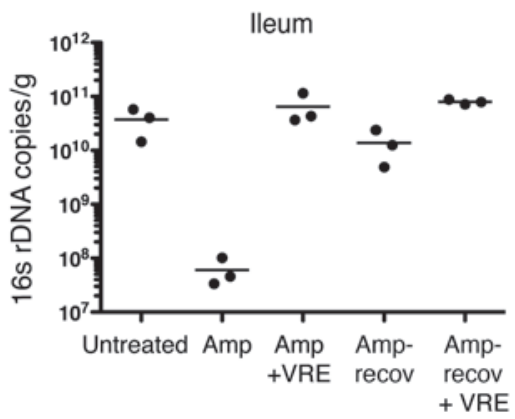

\section{Taxonomy}

VRE
Other taxonomy
Unclassified bacteria
Coriobacteriaceae
Enterobacteriaceae
Unclassified Bacteroidetes
Unclassified Bacteroidales
Unclassified Firmicutes
Unclassified Clostridiales
Clostridiaceae
Lachnospiraceae
Peptostreptococcaceae
Ruminococcaceae
Staphylococcaceae
Bacillaceae
Enterococcaceae (not VRE)
Streptococcaceae
Lactobacillaceae
Erysipelotrichaceae

\section{Figure 5}

Ampicillin treatment promotes VRE intestinal colonization in mice. (A) The number of VRE CFUs in the small intestine or cecum of untreated mice, mice treated for 1 week with ampicillin before infection and kept on ampicillin after VRE infection, or mice switched back to antibiotic-free water 1 day after infection and allowed to recover. Mice were infected with $10^{8} \mathrm{CFUs}$. Samples were harvested on different days after VRE infection. $n \geq 6$ mice per group and day, except day 15 ampicillin $(n=3)$. (B) Phylogenetic classification of $16 S$ rDNA frequencies or (C) number of $16 \mathrm{~s}$ rDNA copies in the ileum and cecum of untreated mice as well as ampicillin-treated mice, infected or not with VRE after 1 week of treatment, and either treated for 8 days or allowed to recover starting at 1 day after infection. The most predominant bacterial populations identified are color coded as indicated. Each bar represents the microbiota composition of an individual mouse. the variation observed between samples (Figure 6A), which indicated that the microbiota of untreated mice contain similar bacterial lineages. Antibiotic-treated mice, at all time points following cessation of ampicillin administration, were distinct from untreated mice (Figure 6A), which indicates that antibiotic treatment produces long-term changes in the microbiota. Analysis of the microbiota of untreated mice and mice that recovered from ampicillin treatment (Figure 6B) confirmed our previous finding (Figure 2) that some bacterial populations, such as the Lactobacillaceae and the Bacteroidetes, did not recover, and demonstrated that this loss persisted for 8 weeks. In contrast, other bacterial populations, such as Clostridiales Incertea Sedis XIV, became major contributors of the new microbial consortium. Most antibiotic-treated mice had increased representation of the Enterococcaceae family and, in a smaller number of mice, an increase in the Clostridiaceae family (Figure 6B). More detailed analysis of these sequences revealed that these bacteria belonged to the genera Enterococcus and Clostridium (data not shown).

To determine whether the long-term effects of antibiotic treatment on the intestinal microbiota increase susceptibility to VRE coloni- 
A

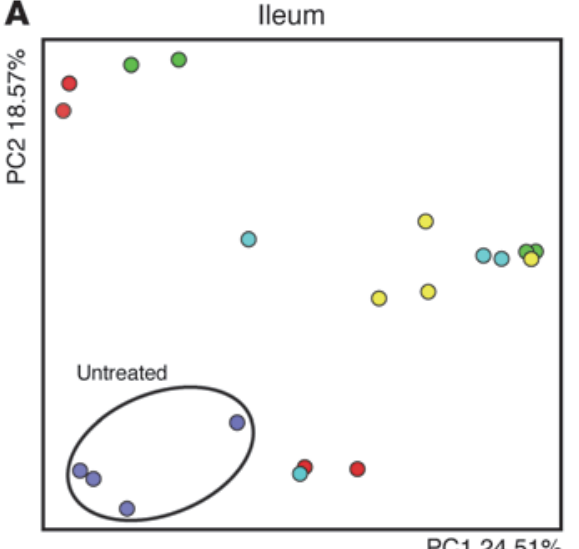

PC1 $24.51 \%$

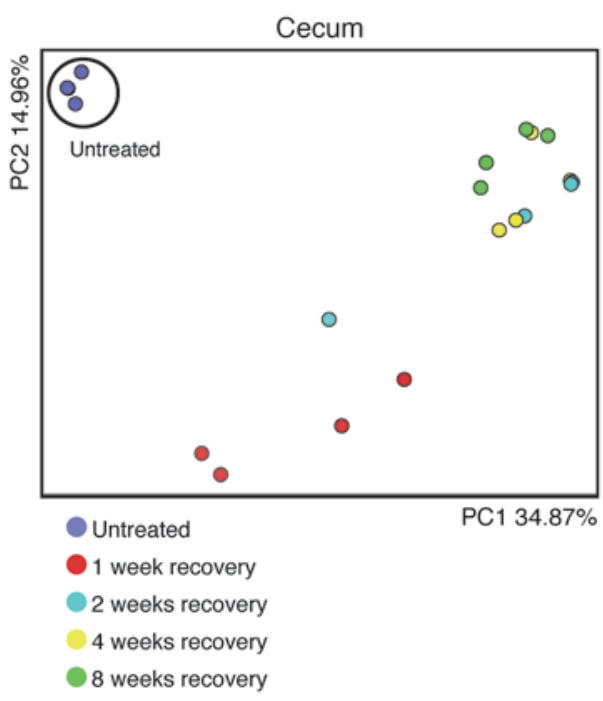

B
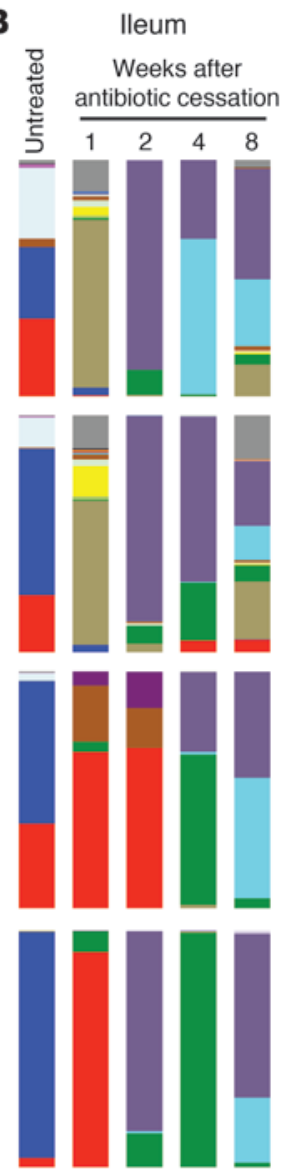

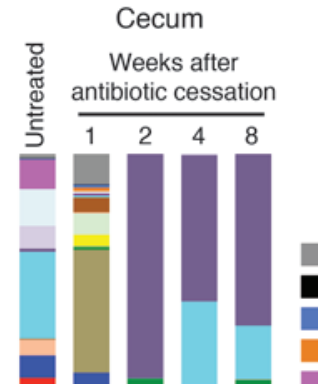

Taxonomy

Other taxonomy

Unclassifed bacteria

Coriobacteriaceae

Enterobacteriaceae

Unclassified Bacteroidetes

Unclassified Firmicutes

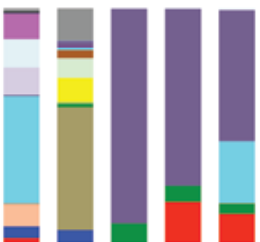

Unclassified Clostridiales

Clostridiales Incertea Sedis XIV

Clostridiaceae

Lachnospiraceae

Peptostreptococcaceae

Ruminococcaceae

Leuconostocaceae

Staphylococcaceae

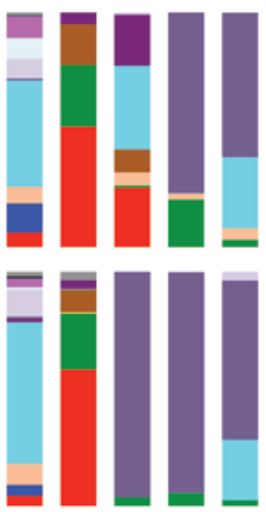

Bacillaceae

Enterococcaceae

Streptococcaceae

- Lactobacillaceae

Erysipelotrichaceae

\section{Figure 6}

Incomplete recovery of the microbiota after ampicillin treatment. Four groups of mice were treated with ampicillin and allowed to recover from ampicillin treatment. Each group of mice was housed in a separate cage. 1 mouse per cage was euthanized at weeks 1,2, 4, or 8 after antibiotic withdrawal, and the ileum and cecum was harvested for microbiota analysis. As controls, 4 mice were euthanized before antibiotic treatment. (A) Unweighted UniFrac analysis of ileum or cecum samples. Each point represents the microbiota of an individual mouse. (B) Phylogenetic classification of 16S rDNA frequencies in the ileum and cecum. Each bar represents the microbiota of an individual mouse. The most predominant bacterial populations identified are color coded as indicated.

zation, mice were treated with ampicillin, allowed to recover from antibiotic treatment for 2 or 4 weeks, and were then infected with $10^{8}$ CFUs of VRE. Consistent with our previous findings (Figure 5), mice that were infected before cessation of ampicillin treatment were densely colonized with VRE 8 days after infection, whereas VRE was completely cleared in most of the untreated mice 8 days after infection (Figure 7). Mice that had recovered from ampicillin treatment for either 2 or 4 weeks prior to VRE inoculation were heavily colonized with VRE 8 days after infection (Figure 7). The density of VRE colonization was less in mice that had recovered from antibiotic treatment than in mice that were still being treated with ampicillin at the time of VRE inoculation. On the other hand, the density of VRE colonization of the cecum was 10,000- to 100,000-fold higher in previously treated mice than in mice that had never received antibiotics. This result indicates that bacterial populations that suppress VRE colonization are lost after an 8-day course of ampicillin and do not recover for up to 2 months after cessation of antibiotic treatment.
Antibiotic effect on VRE intestinal colonization in bumans. VRE is one of the most important causes of bacteremia in hospitalized patients following antibiotic treatment, particularly in cancer patients undergoing chemotherapy and/or allo-HSCT $(36,37)$. Because our experiments with mice demonstrated that antibiotic treatment can result in overwhelming colonization with antibioticresistant microbes, we examined intestinal bacterial colonization in patients undergoing allo-HSCT. Stool samples were collected from these patients upon hospital admission and periodically during the transplant course. For this analysis, we included only those patients from whom we had obtained fecal samples during the first 2 weeks after transplantation. Fecal samples from 2 patients who developed VRE bacteremia during hospitalization and 3 who did not were analyzed by $16 \mathrm{~S}$ rDNA sequencing (Figure 8). Clinical information from patients A-E is shown in Supplemental Table 1. All patients received antibiotics during their hospitalization, for prophylaxis during the period of neutropenia and/or as therapy for 

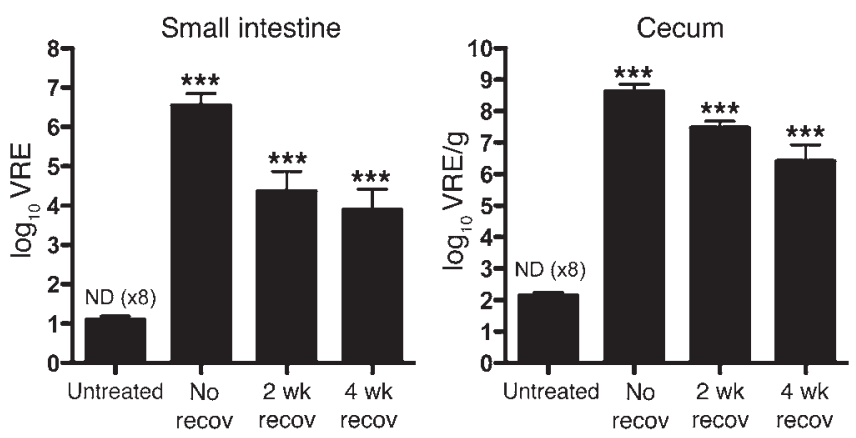

fever or other signs of infection (Supplemental Figure 3). Patients $A$ and $B$ developed VRE bacteremia, whereas patients $\mathrm{C}-\mathrm{E}$ did not. The microbiota of patient A changed dramatically during hospitalization (Figure 8), but eventually bacteria belonging to the genus Enterococcus became the dominant organism (>97\%). This shift preceded the detection of VRE bacteremia by 18 days. Patient B had a diverse fecal microbiota upon hospital admission (Figure 8); 7 days later, after administration of vancomycin and ciprofloxacin, bacteria belonging to the Enterococcus genus expanded dramatically, dominating the microbiota at greater than $98 \%$ frequency. This patient developed VRE bacteremia 3 days after this dramatic shift in the intestinal microbiota. VRE bacteremia persisted in this patient for 18 days and correlated with continued predominant colonization of the intestine with Enterococcus. To determine whether the Enterococci in the fecal samples were the same as those identified in the blood culture, we compared the $16 \mathrm{~s}$ rDNA sequences. The predominant sequence identified by pyrosequencing of fecal samples was identical to the sequence obtained by $16 \mathrm{~s}$ rDNA amplification of the VRE obtained from the blood culture. qPCR of van A, which confers vancomycin resistance, confirmed that the Entero-

\section{Figure 7}

Incomplete recovery of the intestinal microbiota allows VRE intestinal colonization. The number of VRE CFUs was determined 8 days after infection in the small intestine or cecum of untreated mice, mice treated with ampicillin for 1 week before infection and switched back to antibiotic-free water 1 day after infection (i.e., no recovery time), and mice treated with ampicillin for 1 week and allowed to recover for 2 or 4 weeks from ampicillin treatment before infection. If VRE was not detected (ND), the limit of detection was considered to be the number of CFUs in the sample. $n=9$ per group, except 2-week recovery $(n=10)$. ${ }^{* * *} P<0.001$ versus untreated, 1 -way ANOVA with Bonferroni correction on the log-transformed CFU values.

cocci identified in feces were VRE and similar to the isolates from blood cultures (Supplemental Figure 4). The 3 allo-HSCT patients that did not develop a VRE bacteremia did not develop VRE predominance in the feces, even though patient $\mathrm{C}$ was positive for VRE by routine screening rectal swab culture (Supplemental Table 1). Nevertheless, marked shifts in the microbiota were documented over the period of study in these patients (Figure 8).

\section{Discussion}

Because the majority of microbes colonizing the mammalian intestine are not readily cultivated in the laboratory (4), conventional culture techniques have not been able to demonstrate intestinal domination by pathogenic bacteria. Our studies using $16 \mathrm{~s}$ rDNA sequencing approaches provide an unbiased view of the intestinal microbiota and demonstrate the remarkable shifts and losses of microbial diversity associated with antibiotic therapy and colonization with antibiotic-resistant microbes. We found that vancomycin and MNV treatment led to sporadic expansion of Enterobacteriaceae, demonstrating that antibiotic-induced alterations of the microbiota can result in overwhelming expansion of otherwise rare

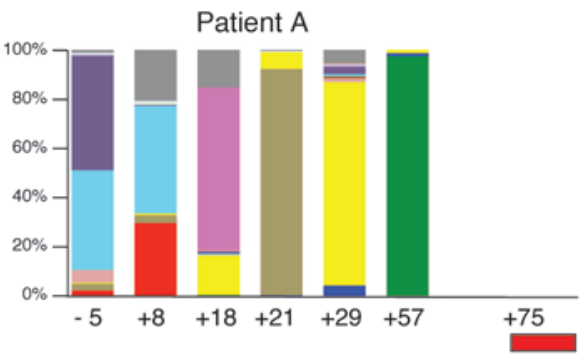

Patient C

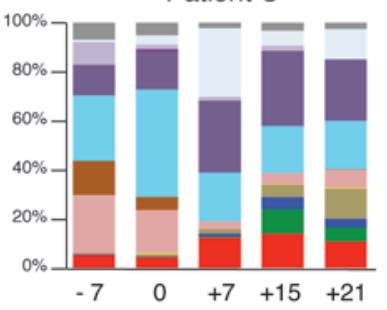

Patient B

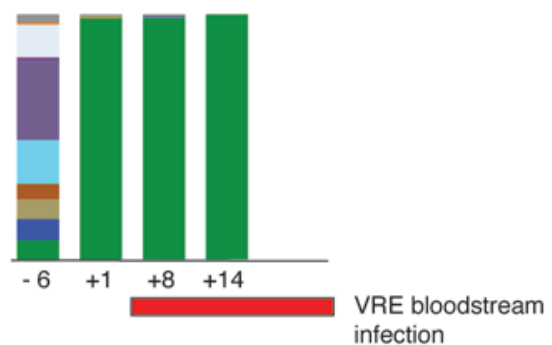

Patient D

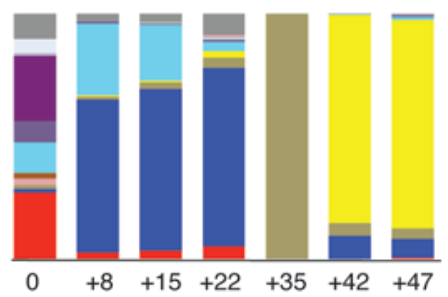
infection

Patient E

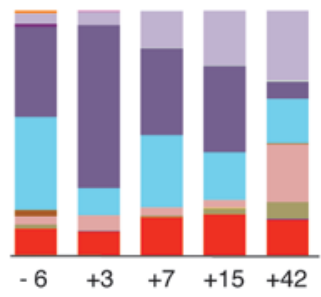

\section{Taxonomy}

Other taxonomy

Porphyromonadaceae

Enterobacteriaceae

Unclassified Firmicutes

Unclassified Clostridiales

Veillonellaceae

Eubacteriaceae

Clostridiales Incertea Sedis XIV

Lachnospiraceae

Peptostreptococcaceae

Ruminococcaceae

Staphylococcaceae

Streptococcaceae

Lactobacillaceae

Enterococcus

Erysipelotrichaceae

Figure 8

VRE dominates the intestinal microbiota in humans prior to invading the bloodstream. Phylogenetic classification of 16S rDNA frequencies in stool samples collected from allo-HSCT patients. Samples were collected upon hospital admission and periodically during the transplant course; 5 patients were studied. Each bar represents the microbiota of 1 stool sample. The timing of sample collection relative to the day of transplant (day 0 ) is indicated below each bar. The most predominant bacterial populations identified are color coded as indicated. The timing of VRE bloodstream infection relative to analyses of the microbiota for patients $A$ and $B$ is indicated by red horizontal bars. 
members of the intestinal consortium. The effects of antibiotic treatment on the microbiota can be complex, with some unexpected results. For example, treatment with vancomycin, an antibiotic that selectively targets Gram-positive bacteria, essentially eliminated the Gram-negative phylum Bacteroidetes from the cecum. Discontinuation of all 3 antibiotic regimens also resulted in a loss of Lactobacillaceae. Other bacterial genera and families, such as Clostridium, Enterococcus, and Enterobacteriaceae, increased in frequency following recovery from antibiotic therapy. Disruption of the microbiota enabled VRE to undergo dramatic expansion and to dominate the microbial consortium of the ileum and cecum. This type of microbial domination was not restricted to experimental mice, but was also found to occur in hospitalized humans exposed to antibiotics.

The major components of the intestinal microbial flora are the bacterial phyla Bacteroidetes and Firmicutes $(4,6,8)$, which are known to influence each other. Bacteria belonging to the Firmicutes phylum, such as Bifidobacterium longum, alter the expression of glycoside hydrolases in Bacteroidetes thetaiotaomicron, a species belonging to the Bacteroidetes phylum (38). Our finding that vancomycin treatment caused a loss of bacteria belonging to the Bacteroidetes phylum suggests that survival of Bacteroidetes in the gut depends on the integrity of Firmicutes populations. Our results are consistent with the findings of Sekirov and coworkers, who used a fluorescent in situ hybridization approach to show decreased prevalence of bacteria belonging to the Bacteroidetes phylum and expansion of $\gamma$-proteobacteria, which includes Enterobacteriaceae, during vancomycin treatment (28). Along similar lines, administration of cefoperazone, a broad-spectrum antibiotic, results in depletion of Bacteroidetes and expansion of Firmicutes after cessation of antibiotic treatment (29). Antibiotic-induced losses of Bacteroidetes may have implications for the development of obesity (9) or the control of intestinal inflammatory diseases (39). Loss of Lactobacilli may adversely affect the establishment of immune tolerance (40). Further studies of antibioticinduced changes on the intestinal microbiota may provide insights into the pathogenesis of obesity and inflammatory bowel disease.

We show here that VRE dominated the microbiota after antibiotic treatment both in mice and in humans. The complete displacement of the normal microbiota may also occur following infection with other bacteria. In fact, we observed domination of the microbiota by the families Staphylococcaceae and Streptococcaceae in 2 of the 5 allo-HSCT patients (Figure 8), and previous studies in mice have shown that Salmonella enterica can dominate the microbiota after streptomycin treatment (41).

Our finding that VRE persisted following cessation of antibiotic treatment contrasts with the resistance of untreated mice to colonization with VRE. Although it is possible that VRE actively suppresses microbiota-induced innate immune defense in the intestine, our measurements of antimicrobial peptide expression did not support this hypothesis (data not shown). Consistent with previous studies (29), we found that some bacterial populations did not recover after antibiotic withdrawal. It is likely that some of these bacterial populations suppress VRE colonization, and their loss enables VRE to persist for long periods of time in the intestine. Our finding that mice that recovered from antibiotic treatment were more susceptible to VRE colonization is consistent with this hypothesis. Similar to VRE, other bacteria, such as Salmonella, persist in the intestine for long periods of time (42). Moreover, as with VRE, mice that recover from antibiotic treatment are more susceptible to Salmonella intestinal colonization (30). Identifying which commensal bacterial species are capable of suppressing intestinal colonization by these impor- tant pathogens may provide new approaches to diminish long-term intestinal carriage and development of systemic infection.

Our studies demonstrate the fragility of the intestinal microbiota in experimental mice and in humans undergoing allo-HSCT. Previous studies characterizing the microbiota of 3 healthy individuals treated with ciprofloxacin demonstrated a loss of diversity, but did not reveal overwhelming intestinal colonization by Enterococci or Enterobacteriaceae (8). Our results characterizing the microbiota of hospitalized allo-HSCT patients contrast with this study of healthy volunteers. The reasons for these differences are likely complex and multifactorial, including the dose, duration, and spectrum of administered antibiotics, the immune status of the host, the chemotherapeutic regimen administered prior to transplantation, the integrity of the intestinal epithelium, and the potential exposure of hospitalized patients to highly antibiotic-resistant bacteria. Nevertheless, the patients we have investigated represent a population affected by infections caused by increasingly prevalent antibiotic-resistant microbes. Understanding the complex microbial populations inhabiting mucosal surfaces and determining their composition using high-throughput DNA sequencing approaches will increase our ability to identify patients at risk for developing invasive bloodstream infections and potentially, by judicious administration of probiotic microbes, allow us to prevent mucosal domination by microbes known to cause invasive infections.

\section{Methods}

Mouse models and housing conditions. Experiments were done with 7-week-old C57BL/6J mice purchased from Jackson Laboratories and housed with irradiated food and provided with acidified water. For experiments involving antibiotic treatment, mice were treated for 1 week with drinking water containing either ampicillin $(1 \mathrm{~g} / \mathrm{l})$, vancomycin $(1 \mathrm{~g} / \mathrm{l})$, or the MNV antibiotic cocktail containing vancomycin $(1 \mathrm{~g} / \mathrm{l})$, metronidazole $(1 \mathrm{~g} / \mathrm{l})$, and neomycin $(0.5 \mathrm{~g} / \mathrm{l})$. For the microbiota recovery experiment after ampicillin cessation shown in Figure 6, and for the experimental infections with VRE, mice were treated with ampicillin $(0.5 \mathrm{~g} / \mathrm{l})$ in their drinking water, which was changed every 3 days. All animal studies were performed in compliance with Memorial Sloan-Kettering institutional guidelines and approved by the institution's IACUC.

VRE infection. Mice were infected by means of gavage with $10^{8} \mathrm{CFUs}$ of VRE (stock no. 700221; ATCC). Bacterial counts were determined by plating serial dilutions of the small intestine and cecal contents on EnterococcoseI agar plates (Difco) with vancomycin ( $8 \mu \mathrm{g} / \mathrm{ml}$; Sigma-Aldrich). VRE colonies were identified by appearance and confirmed by Gram staining. The limit of detection was 10 VRE CFUs in the small intestine samples and 100 VRE CFUs in the cecum samples.

Sample collection and DNA extraction. Fresh stool pellets were obtained before mice were euthanized. Immediately after the mice were euthanized, the contents of the cecum and from the distal $3 \mathrm{~cm}$ of the small intestine (ileum), excluding the last $1 \mathrm{~cm}$ proximal to the cecum, were recovered by manual extrusion. The $3 \mathrm{~cm}$ of the ileum were flushed twice with PBS and saved for DNA extraction from the ileum wall. All samples were snapfrozen in liquid nitrogen prior to storage at $-80^{\circ} \mathrm{C}$. Except for the ileum wall (see below), DNA was extracted using a phenol-chloroform extraction technique with mechanical disruption (bead-beating) based on a previously described protocol (43). Briefly, a frozen aliquot $(-100 \mathrm{mg})$ of each sample was suspended, while frozen, in a solution containing $500 \mu \mathrm{l}$ of extraction buffer (200 mM Tris, pH 8.0; $200 \mathrm{mM} \mathrm{NaCl}$; and $20 \mathrm{mM} \mathrm{EDTA}$ ), $210 \mu \mathrm{l}$ of $20 \%$ SDS, $500 \mu \mathrm{l}$ of phenol/chloroform/isoamyl alcohol (25:24:1), and 500 $\mu \mathrm{l}$ of 0.1 -mm-diameter zirconia/silica beads (BioSpec Products). Microbial cells were lysed by mechanical disruption with a bead beater (BioSpec Prod- 
ucts) for 2 minutes, after which 2 rounds of phenol/chloroform/isoamyl alcohol extraction were performed. DNA was precipitated with ethanol and resuspended in $50 \mu \mathrm{l}$ of TE buffer with $100 \mu \mathrm{g} / \mathrm{ml} \mathrm{RNase.} \mathrm{Fecal} \mathrm{human}$ samples were stored at $-80^{\circ} \mathrm{C}$ prior to DNA isolation. DNA was extracted essentially as described above, except that the isolated DNA was subjected to additional purification with QIAamp mini spin columns (Qiagen). Fecal samples from patients were obtained after their full consent under IRBapproved MSKCC protocol 06-107A.

Total genomic DNA was extracted from the ileum wall samples using a combination of the QIAamp DNA isolation kit (Qiagen) and a bead-beater method based on a previously described protocol (44). Briefly, ileum wall fragments were lysed in $180 \mu \mathrm{l}$ of QIAamp ATL buffer and $20 \mu \mathrm{l}$ of proteinase $\mathrm{K}$ for 1 hour at $56^{\circ} \mathrm{C}$. Zirconia/silica beads $(0.1 \mathrm{~mm}$ diameter) were added, and samples were homogenized in a bead beater (BioSpec Products) for 2 minutes and incubated for an additional hour at $56^{\circ} \mathrm{C} .4 \mu \mathrm{l}$ of RNase $\mathrm{A}(100 \mathrm{mg} / \mathrm{ml})$ and $200 \mu \mathrm{l}$ of AL buffer were added to the lysate, and samples were incubated for 30 minutes at $70^{\circ} \mathrm{C}$. After the addition of $200 \mu \mathrm{l}$ absolute ethanol, lysates were purified over a QIAamp column as specified by the manufacturer.

V2 16S rRNA amplification and 454/pyrosequencing. For each sample, 3 replicate 25- $\mu$ l PCRs were performed, each containing $50 \mathrm{ng}$ of purified DNA, $0.2 \mathrm{mM}$ dNTPs, $1.5 \mathrm{mM} \mathrm{MgCl}_{2}, 1.25$ U Platinum Taq DNA polymerase, $2.5 \mu \mathrm{l}$ of $10 \times$ PCR buffer, and $0.2 \mu \mathrm{M}$ of each primer designed to amplify the $\mathrm{V} 2$ region as previously described (43): a modified primer $8 \mathrm{~F}$ (5'-GCCTTGCCAGCCCGCTCAGAGAGTTTGATCCTGGCTCAG-3'), composite of 454 primer $\mathrm{B}$ (underline) and the universal bacterial primer $8 \mathrm{~F}$ (italics); and the modified primer 338R (5'-GCCTCCCTCGCGCCATCAGNNNNNNNNNNTGCTGCCTCCCGTAGGAGT-3') composite of 454 primer A (underline), a unique 10-base barcode, and the broad-range bacterial primer 338R (italics). When the concentration of DNA was low, such as in the samples from mice treated with antibiotics, $0.5 \mu \mathrm{l}$ of DNA was used in the PCR reaction. Cycling conditions were $94^{\circ} \mathrm{C}$ for 3 minutes, followed by 25 cycles (cecum and fecal samples), 28 cycles (ileum content samples), 30 cycles (ileum wall samples), and 35 cycles (samples with low bacterial density) of $94^{\circ} \mathrm{C}$ for 30 seconds, $52^{\circ} \mathrm{C}$ for 30 seconds, and $72^{\circ} \mathrm{C}$ for $1 \mathrm{~min}$ ute. Replicate PCRs were pooled, and amplicons were purified using the Qiaquick PCR Purification Kit (Qiagen). PCR products were sequenced on a 454 GS FLX platform following the 454 Roche recommended procedures.

Sequence analysis. The sequences reported herein have been deposited in the NCBI Short Read Archive database (accession no. SRA024507). Sequences were converted to standard FASTA format using Vendor 454 software. Sequences shorter than $200 \mathrm{bp}$, longer than $300 \mathrm{bp}$, with undetermined bases, a 454 sequence quality average below 25 , with no exact match to the forward primer or a barcode, or that did not aligned with the $\mathrm{V} 2$ region were not included in the analysis. Sequences were grouped into OTUs using MOTHUR (45). Sequences were aligned, using as template the Silva reference alignment (46) and the Needleman-Wunsch algorithm with default scoring options. Sequence distances were calculated with MOTHUR. To avoid overestimating distances between 454 tag sequences from the rapidly diverging variable regions, as suggested by Sogin and coworkers (47), we treated gaps of any length as a single evolutionary event or mismatch and ignored terminal gaps. Sequences were clustered into OTUs using the furthest-neighbor algorithm. Sequences with distance-based similarity of $97 \%$ or greater were assigned to the same OTU. Taxonomic assignments for a representative sequence from each OTU were obtained using the RDP Classifier (version 10) with the bootstrap cutoff $50 \%$, recommended for sequences shorter than 250 bps (48). The percent of a specific taxon in a sample was calculated by dividing the number of counts of all the OTUs present in a sample that were classified as that taxon by the total number of counts in the sample. In order to determine the proportion of VRE in a murine sample, the pyrosequencing reads were subjected to BLAST analysis against a reference V2 VRE sequence obtained from a PCR product spanning the V2 region using the VRE strain ATCC 700211 as a template. BLAST hits with $\geq 98 \%$ identity and $\geq 98 \%$ coverage were considered to be VREs.

Tree building and UniFrac clustering for PCA analysis. A representative sequence from each OTU was aligned using MOTHUR (45). The PH Lane mask was used to screen out hypervariable regions after alignment. A phylogenetic tree was inferred using the alignment generate and FastTree (49). Unweighted UniFrac (35) was run using the resulting tree. PCA was performed on the resulting matrix of distances between each pair of samples.

Quantification of intestinal microbiota density by qPCR. qPCR was performed on DNA extracted from the cecal and ileum murine contents using DyNAmo SYBR Green qPCR kit (Finnzymes) and $0.2 \mu \mathrm{M}$ of the universal bacterial primer $8 \mathrm{~F}\left(5^{\prime}\right.$-AGAGTTTGATCCTGGCTCAG) and the broad-range bacterial primer 338R (5'-TGCTGCCTCCCGTAGGAGT-3'). Standard curves were prepared by serial dilution of the PCR blunt vector (Invitrogen) containing 1 copy of the $16 \mathrm{~s}$ rRNA gene. Cycling conditions were $95^{\circ} \mathrm{C}$ for 10 minutes followed by 40 cycles of $95^{\circ} \mathrm{C}$ for 30 seconds, $52^{\circ} \mathrm{C}$ for 30 seconds, and $72^{\circ} \mathrm{C}$ for 1 minute.

Quantification of the van A gene by $q$ PCR. Quantitative PCR was performed on DNA extracted from human fecal samples as described above using the Step One Plus Real Time PCR system (Applied Biosystems). qPCR was performed using $12.5 \mu \mathrm{l}$ of $2 \times$ brilliant II qPCR master mix (Stratagene) and $0.4 \mu \mathrm{M}$ of the specific primers for the van $A$ gene: forward, $5^{\prime}$-AATCGGCAAGACAATATGAC; reverse, 5' -ACCTCGCCAACAACTAACGC; FAMlabeled probe, 5'd FAM-GGACGTGTGAGGTCGGTTGTGCGGTATACGTCC-BHQ-1 3'. Standard curves for the van A gene were prepared from serial dilutions of the VRE strain ATCC 700221. The number of van $A$ gene copies was normalized to the number of $16 \mathrm{~s}$ rDNA copies that were quantified as described above. Results are expressed relative to the van $A$ gene levels from a pure culture of the VRE strain isolated from the blood culture (assigned as 100\%).

Statistics. Statistical analysis of the number of $16 \mathrm{~s}$ rRNA copies and the number of VRE CFUs was performed using 1-way ANOVA with Bonferroni correction and Prism software. $P$ values less than 0.05 were considered to be significant. To compute which bacterial taxa had statistically differential V2 16s rDNA counts between treated and untreated mice, we used the edgeR package from the Bioconductor Libraries (www.bioconductor.org) for the $R$ statistical system. The Moderated negative binomial model was used, which extends the Poisson model to account for dispersion in the variance (50). $P$ values were computed using the Fisher exact test, and the false discovery rate method was used to correct for multiple testing. The final results were filtered for $P$ values less than 0.05 , fold change of 2 or greater, and minimum 10 counts in the normalized data.

\section{Acknowledgments}

This work was supported by NIH grants RO1-AI042135 and PO1CA023766 to E.G. Pamer, RO1-CA107096 to M.R.M. van den Brink, a postdoctoral fellowship award from the Cancer Research Institute to C. Ubeda, and funds from the Lucille Castori Center for Microbes, Inflammation, and Cancer.

Received for publication June 3, 2010, and accepted in revised form September 15, 2010.

Address correspondence to: Eric G. Pamer or Carles Ubeda, Laboratory of Antimicrobial Immunity, Infectious Disease Service, Department of Medicine, Memorial Sloan-Kettering Cancer Center, 1275 York Avenue, New York, New York 10065, USA. Phone: 646.888.2361; Fax: 646.422.0502; E-mail: pamere@mskcc.org (E.G. Pamer); ubedamoc@mskcc.org (C. Ubeda). 
1. Donskey CJ. Antibiotic regimens and intestinal colonization with antibiotic-resistant gram-negative bacilli. Clin Infect Dis. 2006;43(suppl 2):S62-S69.

2. Rice LB. Antimicrobial resistance in gram-positive bacteria. Am J Med. 2006;119(6 suppl 1):S11-S19.

3. Bartlett JG. Narrative review: the new epidemic of Clostridium difficile-associated enteric disease. Ann Intern Med. 2006;145(10):758-764.

4. Eckburg PB, et al. Diversity of the human intestinal microbial flora. Science. 2005;308(5728):1635-1638.

5. Backhed F, Ley RE, Sonnenburg JL, Peterson DA, Gordon JI. Host-bacterial mutualism in the human intestine. Science. 2005;307(5717):1915-1920.

6. Ley RE, Peterson DA, Gordon JI. Ecological and evolutionary forces shaping microbial diversity in the human intestine. Cell. 2006;124(4):837-848.

7. Versalovic J, Relman D. How bacterial communities expand functional repertoires. PLoS Biol. 2006;4(12):e430.

8. Dethlefsen L, Huse S, Sogin ML, Relman DA. The pervasive effects of an antibiotic on the human gut microbiota, as revealed by deep $16 \mathrm{~S}$ rRNA sequencing. PLoS Biol. 2008;6(11):e280.

9. Ley RE, Turnbaugh PJ, Klein S, Gordon JI. Microbial ecology: human gut microbes associated with obesity. Nature. 2006;444(7122):1022-1023.

10. Hooper LV, Midtvedt T, Gordon JI. How hostmicrobial interactions shape the nutrient environment of the mammalian intestine. Annu Rev Nutr. 2002;22:283-307.

11. Backhed F, et al. The gut microbiota as an environmental factor that regulates fat storage. Proc Natl Acad Sci U S A. 2004;101(44):15718-15723.

12. Stappenbeck TS, Hooper LV, Gordon JI. Developmental regulation of intestinal angiogenesis by indigenous microbes via Paneth cells. Proc Natl Acad Sci US A. 2002;99(24):15451-15455.

13. Rakoff-Nahoum S, Paglino J, Eslami-Varzaneh F, Edberg S, Medzhitov R. Recognition of commensal microflora by toll-like receptors is required for intestinal homeostasis. Cell. 2004;118(2):229-241.

14. Cebra JJ. Influences of microbiota on intestinal immune system development. Am J Clin Nutr. 1999;69(5):1046S-1051S.

15. Vollaard EJ, Clasener HA. Colonization resistance. Antimicrob Agents Chemother. 1994;38(3):409-414.

16. Pamer EG. Immune responses to commensal and environmental microbes. Nat Immunol. 2007; 8(11):1173-1178.

17. Brandl K, et al. Vancomycin-resistant enterococci exploit antibiotic-induced innate immune deficits. Nature. 2008;455(7214):804-807.

18. Abreu MT, Thomas LS, Arnold ET, Lukasek K, Michelsen KS, Arditi M. TLR signaling at the intestinal epithelial interface. J Endotoxin Res. 2003 9(5):322-330.

19. Gewirtz AT, Navas TA, Lyons S, Godowski PJ, Madara JL. Cutting edge: bacterial flagellin activates basolaterally expressed TLR5 to induce epithelial proinflammatory gene expression. J Immunol. 2001;167(4):1882-1885.

20. Kobayashi KS, et al. Nod2-dependent regulation of innate and adaptive immunity in the intestinal tract. Science. 2005;307(5710):731-734.

21. Rakoff-Nahoum S, Medzhitov R. Role of the innate immune system and host-commensal mutualism. Curr Top Microbiol Immunol. 2006;308:1-18.

22. Ayabe T, Satchell DP, Wilson CL, Parks WC, Selsted ME, Ouellette AJ. Secretion of microbicidal alphadefensins by intestinal Paneth cells in response to bacteria. Nat Immunol. 2000;1(2):113-118.

23. Vora P, et al. Beta-defensin-2 expression is regulated by TLR signaling in intestinal epithelial cells. J Immunol. 2004;173(9):5398-5405.

24. Cash HL, Whitham CV, Behrendt CL, Hooper LV. Symbiotic bacteria direct expression of an intestinal bactericidal lectin. Science. 2006 313(5790):1126-1130.

25. Brandl K, Plitas G, Schnabl B, Dematteo RP, Pamer EG. MyD88-mediated signals induce the bactericidal lectin RegIII $\{$ gamma\} and protect mice against intestinal Listeria monocytogenes infection. J Exp Med. 2007;204(8):1891-1900.

26. Vaishnava S, Behrendt CL, Ismail AS, Eckmann L, Hooper LV. Paneth cells directly sense gut commensals and maintain homeostasis at the intestinal host-microbial interface. Proc Natl Acad Sci U S A 2008;105(52):20858-20863.

27. Kinnebrew MA, Ubeda C, Zenewicz LA, Smith N, Flavell RA, Pamer EG. Bacterial flagellin stimulates Toll-like receptor 5-dependent defense against vancomycin-resistant Enterococcus infection. J Infect Dis. 2010;201(4):534-543.

28. Sekirov I, et al. Antibiotic-induced perturbations of the intestinal microbiota alter host susceptibility to enteric infection. Infect Immun. 2008 76(10):4726-4736

29. Antonopoulos DA, Huse SM, Morrison HG, Schmidt TM, Sogin ML, Young VB. Reproducible community dynamics of the gastrointestinal microbiota following antibiotic perturbation. Infect Immun. 2009;77(6):2367-2375.

30. Croswell A, Amir E, Teggatz P, Barman M, Salzman $\mathrm{NH}$. Prolonged impact of antibiotics on intestinal microbial ecology and susceptibility to enteric Salmonella infection. Infect Immun. 2009;77(7):2741-2753.

31. Garner CD, et al. Perturbation of the small intestine microbial ecology by streptomycin alters pathology in a Salmonella enterica serovar typhimurium murine model of infection. Infect Immun. 2009 ; 77(7):2691-2702

32. Lawley TD, et al. Antibiotic treatment of clostridium difficile carrier mice triggers a supershedder state, spore-mediated transmission, and severe disease in immunocompromised hosts. Infect Immun. 2009; 77(9):3661-3669.

33. Hill DA, et al. Metagenomic analyses reveal antibiotic-induced temporal and spatial changes in intestinal microbiota with associated alterations in immune cell homeostasis. Mucosal Immunol. 2010;3(2):148-158

34. Donskey CJ, Hanrahan JA, Hutton RA, Rice LB. Effect of parenteral antibiotic administration on persistence of vancomycin-resistant Enterococcus faecium in the mouse gastrointestinal tract. J Infect Dis. 1999;180(2):384-390.
35. Lozupone C, Hamady M, Knight R. UniFrac--an online tool for comparing microbial community diversity in a phylogenetic context. BMC Bioinformatics. 2006;7:371.

36. Weinstock DM, et al. Colonization, bloodstream infection, and mortality caused by vancomycinresistant enterococcus early after allogeneic hematopoietic stem cell transplant. Biol Blood Marrow Transplant. 2007;13(5):615-621.

37. Zirakzadeh A, Gastineau DA, Mandrekar JN, Burke JP, Johnston PB, Patel R. Vancomycin-resistant enterococcal colonization appears associated with increased mortality among allogeneic hematopoietic stem cell transplant recipients. Bone Marrow Transplant. 2008;41(4):385-392.

38. Sonnenburg JL, Chen CT, Gordon JI. Genomic and metabolic studies of the impact of probiotics on a model gut symbiont and host. PLoS Biol. 2006; 4(12):e413

39. Mazmanian SK, Round JL, Kasper DL. A microbial symbiosis factor prevents intestinal inflammatory disease. Nature. 2008;453(7195):620-625.

40. van Baarlen P, et al. Differential NF-kappaB pathways induction by Lactobacillus plantarum in the duodenum of healthy humans correlating with immune tolerance. Proc Natl Acad Sci U S A. 2009. 106(7):2371-2376.

41. Stecher B, et al. Salmonella enterica serovar typhimurium exploits inflammation to compete with the intestinal microbiota. PLoS Biol. 2007;5(10):2177-2189.

42. Kingsley RA, van Amsterdam K, Kramer N, Baumler AJ. The shdA gene is restricted to serotypes of Salmonella enterica subspecies I and contributes to efficient and prolonged fecal shedding. Infect Immun. 2000;68(5):2720-2727.

43. Turnbaugh PJ, et al. A core gut microbiome in obese and lean twins. Nature. 2009;457(7228):480-484.

44. Bik EM, et al. Molecular analysis of the bacterial microbiota in the human stomach. Proc Natl Acad Sci U S A. 2006;103(3):732-737.

45. Schloss PD, et al. Introducing mothur: open-source, platform-independent, community-supported software for describing and comparing microbial communities. Appl Environ Microbiol. 2009; 75(23):7537-7541.

46. Pruesse E, et al. SILVA: a comprehensive online resource for quality checked and aligned ribosomal RNA sequence data compatible with ARB. Nucleic Acids Res. 2007;35(21):7188-7196.

47. Sogin ML, et al. Microbial diversity in the deep sea and the underexplored "rare biosphere". Proc Natl Acad Sci U S A. 2006;103(32):12115-12120.

48. Wang Q, Garrity GM, Tiedje JM, Cole JR. Naive Bayesian classifier for rapid assignment of rRNA sequences into the new bacterial taxonomy. Appl Environ Microbiol. 2007;73(16):5261-5267.

49. Price MN, Dehal PS, Arkin AP. FastTree: computing large minimum evolution trees with profiles instead of a distance matrix. Mol Biol Evol. 2009; 26(7):1641-1650

50. Robinson MD, Smyth GK. Moderated statistical tests for assessing differences in tag abundance. Bioinformatics. 2007;23(21):2881-2887. 